\title{
Internet of Things Framework for Structural Health Monitoring in Nigeria
}

\author{
${ }^{1}$ Boyinbode O.K., ${ }^{2}$ Oyesanmi F.G., ${ }^{3}$ Obe O.O.,${ }^{4}$ Boyinbode O.F. \\ ${ }^{1}$ Department of Information Technology, Federal University of Technology Akure, Nigeria, \\ okboyinbode@ futa.edu.ng \\ ${ }^{2}$ Department of Computer Science, Federal University of Technology Akure, Nigeria, fiyin001@ gmail.com \\ ${ }^{3}$ Department of Computer Science, Federal University of Technology Akure, oobe@ futa.edu.ng \\ ${ }^{4}$ Directorate of Physical Planning, Federal University of Technology, Akure, Nigeria, ofboyinbode@ futa.edu.ng
}

\begin{abstract}
Internet of Things (IoT) is a paradigm that has been explored in various domains, as it facilitates the automation of many tedious processes with more efficiency and robustness. This technology can be applied to effectively mitigate the rising cases of building collapse in Nigeria. Traditional means of monitoring civil structures and determining their health seems inadequate, expensive and out of reach to many, hence a novel means of Structural Health Monitoring (SHM) which IoT provides. IoT facilitates the monitoring and measurement of several structural parameters with a high degree of precision through sensors while enabling these 'things' to communicate effectively through the designed protocol. It has proved to be a cost-effective alternative to traditional SHM while providing the flexibility of alerting users when there is any damage to the structure. In this paper, an IoT platform for structural integrity evaluation and monitoring in civil buildings was developed using piezoelectric transducers (PZT), some microcontrollers, and a SIM800L. These PZTs were deployed at determined nodes to read data continually while some digital signal processing techniques were used to manipulate these data towards determining the health of the structure being monitored. In the end, a system that reads signals from structures, store the signals on the cloud and notify users if any structural damage arises were developed.
\end{abstract}

Key words: Internet of Things (IoT), Structural Health Monitoring (SHM), Piezoelectric transducers (PZT), Microcontroller (MCU)

\section{INTRODUCTION}

Structures are an indispensable part of life. But these structures need to be properly monitored to ensure that they carry out the purpose of their design continually without constituting a threat to life and public safety. Hence, structural health monitoring (SHM).Structural Monitoring includes the several activities of experts in ensuring that structures (civil and industrial) can still sustain the purpose of their construction. These activities usually involve gathering information about several components of the structure through which informed decisions about its state can be deduced. Wang et al [1] reported that one of the earliest permanent structural monitoring of high-rise buildings dates back to the 1940s. The need to track and measure the progress of structures continually and as a permanent feature gave birth to the term Structural Health Monitoring.

Structural Health Monitoring (SMH) deals with the continuous monitoring of civil and industrial structures (such as buildings, bridges, roads, pipelines, dams, power grids, etc.) towards facilitating public safety while reducing the overhead on maintenance. It involves the use of sensors (smart objects) to acquire information about the alterations in a single part of the whole structure in a noninvasive manner.

Appropriate mathematical methods are thereafter merged with the information obtained from the smart objects which are then used to determine the safety of structures [2]. SHM is a proactive measure to prevent building failure. Building failures manifest in various ways, mostly through damages to key structures such as pillars and columns, foundation, beam, and roof. A major pointer to building defects or failure can be seen through building cracks. Haruna et al, [3] observe that structural cracks often appear on building columns and beams which form the skeleton for buildings. Thus, sensors to monitor civil structures must target these key parts of a building.

The availability of Smart objects (sensors) which can measure and communicate with precision data such as accelerations, humidity, temperature, accelerations, tensile stress, compressive stress, and 
building materials degradation is fundamental to SHM. Internet of Things has enabled these various smart objects to collect, interpret, and store diverse data from structures autonomously, making SHM more robust.

According to $\mathrm{Xu}$ et al, [4],IoT is defined as dynamic global network infrastructure. It has self-configuring capabilities based on standard and interoperable communication protocols with physical and virtual 'things' which have identities, physical attributes and use intelligent interfaces. Thus, they are seamlessly integrated into the information network.

IoT leverages on the permeation of the internet, the multiplicity of internet-enabled devices and several other technologies which make sensing and reporting with a high degree of precision possible, to provide the solution to many problem domains. These networks of devices interact with each other via machine to machine communications, enabling collection and exchange of data which can be used to monitor civil and industrial structures continuously, determining their health and integrity. Hence, IoT can effectively facilitate public safety emergency management and early warning system which can realize the function of Omni-directional monitoring and control, accurate prediction, and efficient disposal [5].

The application of IoT to structural health monitoring has been boosted by the advancement in the development of sensors having the ability to detect vibrations in structures, read the acoustic emissions, take a highly graphical image of structures, etc., to facilitate the evaluation and monitoring processes [6]. An indispensable quality that makes the relationship between IoT and SHM more attractive is the ability to store the information collected from various structures to the cloud, enhancing their accessibility when needed.

IoT has found applications in several domains across the globe and has been explored to a great deal of success both in literature and in industries, with Structural Health Monitoring one of those domains. Regarded as the driver of the fourth commercial wave, the call for its applications is in great demand [7]. It has equally been explored in several fields in Nigeria such domains include agriculture, health, and security. However, little has been done concerning Structural Health Monitoring in Nigeria.

\section{RELATED WORK}

Several IoT and SHM techniques have been examined, explored, and discussed in the literature and the industry to a diverse degree of success. Scuro et al., [2]implemented an advanced SHM technique based on Acoustic Emission (AE) system to estimate the extent of damage in a structure. The method determines the placement of such error and detect the formation of the damage. It was estimated that the values evaluated by the emission of ultrasonic waves in the structure and the acquisition of the eco allow the system to evaluate the presence of damage in structures and their placement while the AE can be used to detect the damage formation. The system design has a reduced battery consumption rate with the capability of continuously monitoring existing and new structures. The downside to this research work is that it is a theoretical work and the accuracy of the system was not tested.

Determined to improve the safety and maintainability of critical structures such as bridges and buildings, Abdelgawad and Yelamarthi [8] researched on how to detect the location of damages on structures and the extent of such damages. In the methodology explored, two piezoelectric sensors were used, as well as a Pro-Trinket microcontroller, NRF transceiver module, Wi-Fi module, and Raspberry Pi. These devices were used to collect data from structures, establish if damage exists in such structures or not, then determine the location and width of such structures before storing such data on the cloud and made assessable remotely from any mobile device. The design and implementation of the IoT SHM platform determined the structural damage, location, and size with high-level precision.

Abdelgawad and Yelamarthi[8] also explored IoT technology to meet the rising demand for a platform that can provide reliable structural health information, predict upcoming accidents, and estimate the useful life span of a structure where such data can be available anytime and anywhere to enhance public safety. The work sought an SHM platform embedded with IoT to determine the size and placement of damage in structures. The data from the platform can be stored and accessed remotely from any mobile device. To achieve this, a crosscorrelation (CC) algorithm was deployed to affirm the similarity between excitation signal, E, at PTZ1, and received signal, $\mathrm{R}$, at PTZ2 attached to the MCU. The damage placement and size were then determined and delivered to the cloud. The design was implemented using a zinc plate which displayed a $1.03 \%$ error for the damage placement and an $8.43 \%$ error for the damage width recorded.

Hsu et al, [9] implemented the design of a WSN suitable for on-field Structure Health Monitoring of real-time data with efficiency over existing sensors. The WSN system can collect vibration acceleration from bridges in real-time and transmit those data packages through the Internet to designated users. The methodology used was the design and 
development of a customized smart device named Super-Node. A sensor node with MSP430 1611 16bits chip as processing unit, UZ2400 wireless radio chip equipped with 802.15 .4 protocol as the transceiver. There were several $\mathrm{I} / \mathrm{O}$ channels also configured with an accelerometer. Built with $\mathrm{C}$, it contains two nodes, the first for data transmission from sensors, the second one is the coordinator in charge of data collection, and passing data packages to the antenna. At implementation, the designed system has the wireless transmission payload reduced by $60 \%$, and the node capacity increased by 3 times: an improvement over existing SHM systems especially the wired and remote methods used by civil engineers.

Concerned about the safety of civil structures, especially first-order tunnels, [10] developed a model for monitoring and assessing their integrity using wireless sensor networks (WSNs). Fiber OS was used to detect the variation of temperature and strain over different parts of the structure. The sensor nodes were also placement deterministically to influence the efficiency of WSN and its operation. In all, the research established that the deployment of sensors for SHM must be deterministic (controlled) rather than random for efficiency and effectiveness. The work identified the various challenges of deploying WSN in a tunnel such as electromagnetic interference and noise.

Lake et al, [11] designed an SHM and notification system for structures such as bridges, buildings, and airframes. Piezoelectric transducers were used as sensors to read data from buildings, an MCU, Raspberry Pi control the sensors and create an excitation signal, receive the data, process the data, and then send it to the user via email or the cloud. Overall, the system designed and implemented a lowcost model for detecting damages and their sizes, if they exist in solid structures with a relatively high degree of accuracy. The research, however, did not reference any expert in the domain of civil/structural/buildings engineering to ascertain the necessary parts of the building that needs to be monitored.

Propelled by the necessity to preserve national building heritage against seismic risk, Zinno et al, [12] designed an SHM model to limit the effects of earthquakes on such structures. The SHM design was developed using IoT sensors with robust mathematical models to read and predict seismic wave movements and the impact on structures. This system designed was able to accurately monitor as well as self-diagnose the structural state of the health of the building and detect if it is dangerous for users. The system developed was however costly.
Lu and Yang [6] explored the use of IoT technologies to ascertain and enhance the safety of transportation infrastructure frequently used, such as railroads, bridges, and ramps. They aimed to take advantage of the rapid advancement in low $\square$ power processors, sensors, wireless communication, and data storage to automate the inspection process of these transport infrastructures. To this end, a specially designed edge sensor devices called Bridge Monitoring Unit (BMU) which could cope with outdoor sensing was used. In all, the design and implementation of a novel IoT system for outdoor monitoring of bridges using rechargeable batteries were achieved. Nevertheless, the use of rechargeable batteries limits the longevity and autonomy of the system designed.

Richardson et al, [13] used Modal Parameters for Structural Health Monitoring which could detect and quantify changes in the physical properties of a structure. The use of modal frequency and damping, together with Shape Difference Indicator, SDI metric as a means of detecting and quantifying changes in structural stiffness was deployed in their methodology. The system designed was called Fault Correlation Tools $\left(\mathrm{FaCTs}^{\mathrm{TM}}\right)$. FaCTs ${ }^{\mathrm{TM}}$ can be used to graphically indicate any change in a specific structural change such as torque, a unique perspective to SHM.

While these various IoT and SHM techniques and more have been deployed on structures, it has been established that IoT can effectively and efficiently monitor and evaluate structures[14]. A major downside despite this success recorded is that there has been no standard agreed upon, and specifications concerning the technologies and tools to deploy in the implementation of these diverse IoT - SHM models. Some of these techniques were adopted to developing an IoT-SHM system that is suitable for the Nigerian domain.

\section{METHODOLOGY}

The IoT SHM platform implemented in this work uses two microcontrollers, four Piezoelectric transducers (PZT), and SIM800L. The piezoelectric transducer is an electro acoustic transducer used or conversion of pressure or mechanical stress into alternating electric force. The four PZTs here are at the sensing layer of this IoT system. They are attached to the structure to be monitored to measure and record lamb waves. The first microcontroller (MCU) used is dsPIC33EP128MC502, an analog to digital converter through which a lamb frequency sound wave was generated to excite one of the transducers (PZT 1) while simultaneously monitoring and reading signals from the receptor sensors (PZT 2 ... 4). The first transducer (PZT1) induces the signal 
towards the receptor transducers (PZT $2 \ldots 4$ ) and choose any wave that has bounced back from the damage that could not reach these receptor transducers, using the pulse-echo technique. The second MCU, ATMEGA328p is responsible for the data retention and IoT publication of this system. The SIM module, SIM800L integrated as part of the IoT system is relay processed data to the cloud server and also send an SMS to designated phone numbers in case any structural damage is noticed[15]. The cloud server used for data visualization for this work is www.thingspeak.com.

The architecture of the system is presented in figure 1 below.

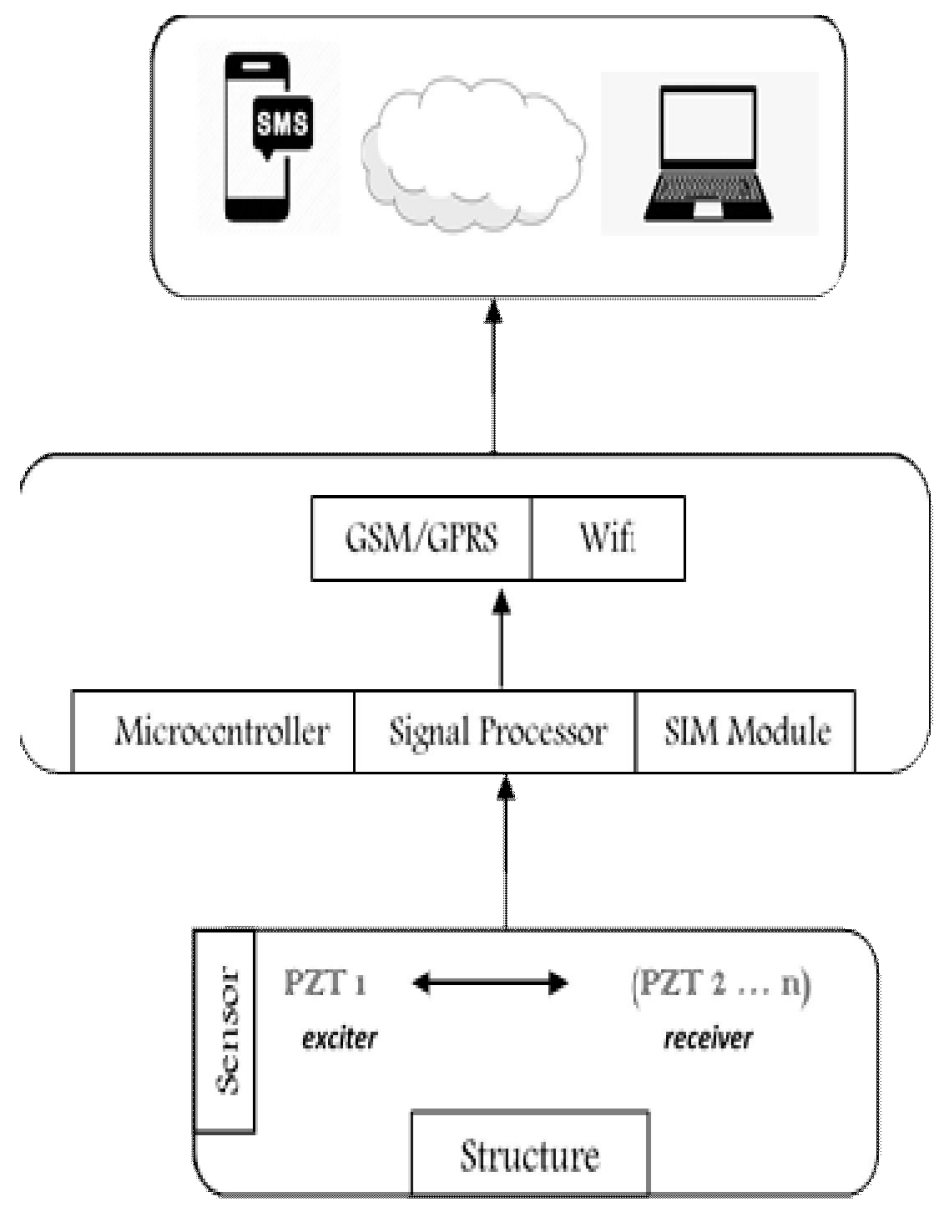

Figure 1: System Architecture

\section{SYSTEM IMPLEMENTATION}

The various components that made up the system have coalesced on a printed circuit board through which an alternating current was supplied to the system. The programming language used was Embedded C, which has a robust library to support the endeavour. The circuit diagram is presented in figure 2 .

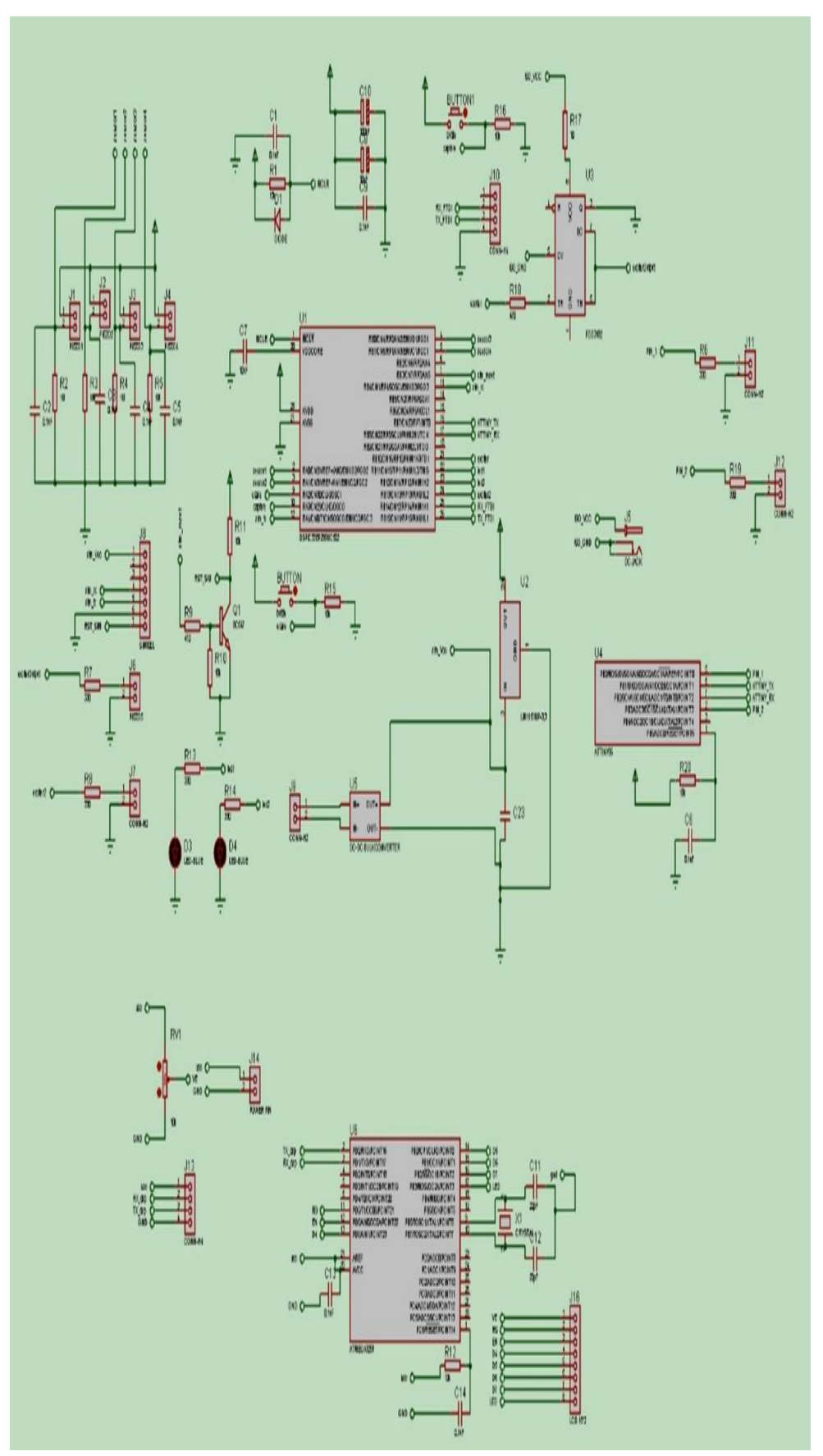

Figure 2: Circuit Diagram

The PZTs were placed at determined nodes on the structure when the system is initiated, the MCU sends an excitation signal which runs for a predetermined amount of time. The system calibrates and records the initial value gotten from the exciter and receiver sensors. These values are stored as reference for the other set of signals that would be generated for the period of evaluation. Thereafter, the time of flight from each sensor node is determined as well as the signal amplitude and the average of each data 
received at intervals. These values would be relative at each node for each period of evaluation. Deviations from the previous values indicate the presence of damage or crack which would be reported to users via a text message.

The choice of a SIM800L over a Wi-Fi chip in this work owes to the wide coverage GSM/GPRS has over Wi-Fi services which has little coverage in Nigeria and costs more. This Subscribers Identification Module (SIM) device, SIM800L, is a GSM/GPRS module, a miniature GSM modem. It replicates the functions of regular cell phones such as SMS, making or receiving phone calls, connecting to the internet through GPRS, TCP/IP, etc. Through this module, data generated from the system are easily transferred to the cloud and users can be notified of the health status of their building via SMS or phone call.The dsPIC33EP128MC502 relays processed data to ATMEGA328p using the UART protocol while SIM800L uses GSM/GPRS technology to relay data to "Thingspeak" server and also alert users through SMS

\section{CONCLUSION}

This IoT system developed for Structural Health Monitoring was able to achieve its aim of monitoring a structure while determining its health status. An SMS was also sent to alert users of the health status of their structure. The system was designed using two microcontrollers, dsPIC33EP128MC502 and ATMEGA328p. The former, an ADC for reading signals from the sensors, the later for data manipulation and publishing. For the sensing layer, piezoelectric transducers were used, a SIM800L subscriber's identification module was used for the data transmission and to facilitate a user's alert system via SMS. Moreover, the data from the sensor nodes are stored on the internet and can be accessed on the webserver via mobile devices or a computer system. This can be used to facilitate a Building Information System.

\section{REFERENCES}

1. Wang M. L., Lynch J. P. \& Sohn H. (2014). Introduction to Sensing for Structural Performance Assessment and Health Monitoring.

2. Scuro C, Lamonaca F., Sciammarella P. F., Carnì D. L., \&Olivito R. (2018). "Internet of things for structural health monitoring". Proc. IEEE Int. Workshop on Metrology for Industry 4.0 and IoT, Apr. 2018.

3. Haruna, Gambo\& Hamza, Umar \&Abdulfatah, A. \& Suleiman, Aminu. (2013). Geotechnical
Investigation into the Causes of Cracks in Building: A Case Study. Electronic Journal of Geotechnical Engineering. 18.2823.

4. Xu, L. D., He, W., \& Li, S. (2014). Internet of Things in Industries: A Survey. IEEE Transactions on Industrial Informatics. IEEE Computer Society. https://doi.org/10.1109/TII.2014.2300753

5. Du, C \& Zhu S. (2012). "Research on urban public safety emergency management early warning system based on technologies for the internet of things”. 2012 International Symposium on Safety Science and Technology. Procedia Engineering 45 (2012) 748 - 754

6. Lu G \& Yang Y.J. (2017). Structural Health Monitoring. Internet of Things and Data Analytics Handbook, First Edition (C) 2017 John Wiley \& Sons, Inc. Published 2017 by John Wiley \& Sons, Inc.

www.wiley.com/go/Geng/IoT_data_analytics_ha ndbook/

7. Noman S. \& Sandhya A. (2017). "Internet of Things: Vision, Application Areas, and Research Challenges". International conference on I-SMAC (IoT in Social, Mobile, Analytics, and Cloud). 978-1-5090-3243-3/17. pp 583-587

8. Abdelgawad, A. \& Yelamarthi, K., (2017). "Internet of Things (IoT) Platform for Structure Health Monitoring". Hindawi Wireless Communications and Mobile Computing Volume 2017, Article ID 6560797. https://doi.org/10.1155/2017/6560797

9. Hsu, Wei-Chih, Chi-Wei Kuo, Wen-Wei Chang, Jhe-Jhao Chang, Yi-Ting Hou, Yi-Chung Lan, Tung-Jung Sung, and Yao-Joe Yang. "A WSN smart medication system." Procedia Engineering 5 (2010): 588-591.

10. Zrelli A. \&Ezzedine T., (2016). "A Comparative Strategies of Node Deployment for Structural Health Monitoring”. Proceedings of 2016 4th International Conference on Control Engineering \& Information Technology (CEIT -2016) Tunisia. Hammamet -December. 16-18. 2016. ISBN: 9781-5090-1055-4 () 2016 IEEE

11. Lake, M., Wernette, J., Khan, T., \&Abdelgawad, A. (2017). Design of a structural health monitoring and notification system. In IEEE International Conference on Electro Information Technology (pp. 321-323). IEEE Computer Society. https://doi.org/10.1109/EIT.2017.8053378

12. Zinno, R., Artese, S., Clausi, G., Magarò, F., Meduri, S., Miceli A., \&Venneri A. (2019). "Structural Health Monitoring (SHM)" Springer International Publishing AG, F. Cicirelli et al. (eds.), The Internet of Things for Smart Urban Ecosystems, Internet of Things, https://doi.org/10.1007/978-3-319-96550-5_10 
13.Richardson, Shawn \& Tyler, Jason \& Schwarz, Brian \&McHargue, Patrick \& Richardson, Mark. (2017). Using Modal Parameters for Structural Health Monitoring. 10.1007/978-3-319-541099_8.

14.Ahmed. A. A. Gad-Elrab, Shereen A. El-aal, Neveen I. Ghali, Afaf A. S. Zaghrout "A Dynamic Genetic-Based Context Modeling Approach in Internet of ThingsEnvironments", International Journal of AdvancedTrends in Computer Science and Engineering Vol. 8, no. 6, pp. 2699-2709, 2019.

https://doi.org/10.30534/ijatcse/2019/03862019,

15.Koduru Suresh1, PVGD Prasad Reddy, PadalaPushkal., "Smart Home Services Using Cloud and Internet of Things", International Journal of Advanced Trends in Computer Science and Engineering, vol. 8, no. 4, pp. 1560-1567, 2019.

https://doi.org/10.30534/ijatcse/2019/79842019 